\section{Módulos interactivos en línea de semiología médica. Una herramienta para estandarizar el aprendizaje clínico}

\author{
ALEJANDRO GONZÁLEZ , BRYAN VARGAS², VICENTE GONZÁLEZ², \\ IGNACIO REYES ${ }^{2}$, ALBERTO SARFATIS ${ }^{1}$
}

\section{Interactive tools to standardize semiology teaching}

Background: The learning process for medical semiology depends on multidisciplinary teaching activities, including simulation tools. These tools should achieve a standardization level aiming at a same level of basic knowledge in each student. Aim: To evaluate an interactive online semiology learning tool. Material and Methods: An interactive online learning method for medical semiology was developed. It focused mainly on physical examination and incorporated audiovisual and self-explanatory elements, to strengthen the acquisition of skills and basic knowledge for each standardized clinical learning simulation session. Subsequently, a satisfaction survey was conducted. Also the performance of students in a clinical examination was compared with that of students of the previous year. Results: Student satisfaction was outstanding, and there was a significant improvement in the performance on the final exam. Conclusions: The use of interactive self-learning online content for medical semiology provides an effective tool to improve student learning.

(Rev Med Chile 2016; 144: 1605-1611)

Key words: Education, Medical; Education, Medical, Undergraduate; Physical Examination; Simulation Training.

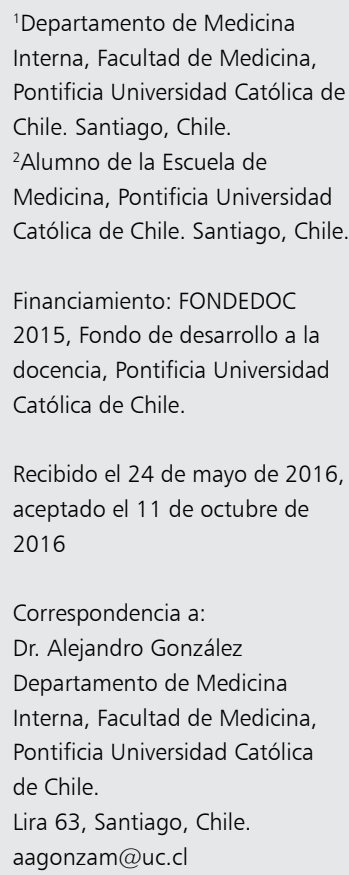

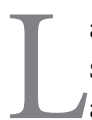
a semiología es la ciencia que estudia los síntomas y signos de las enfermedades ${ }^{1}$. De acuerdo a esta definición, se puede determinar con certeza el carácter multidisciplinario de su enseñanza y aprendizaje, para lo cual se requiere desarrollar habilidades en comunicación, examen físico, interpretación, diagnóstico de los principales síndromes de la medicina ${ }^{2,3}$.

Enmarcados en una sociedad cambiante y que demanda a los médicos competencias y aptitudes sobresalientes, no es de extrañar que estas exigencias se deduzcan, como dicta la lógica, en la implícita necesidad de instituciones y sistemas de enseñanza de excelencia. En la búsqueda de esta excelencia homogénea es que se han desarrollado herramientas que, de manera sistematizada, pudieran facilitar el aprendizaje del alumno de medicina ${ }^{4}$. Dentro de estas herramientas, una de las más novedosas y con mayor potencial de expansión es el aprendizaje en línea. Puede que no resulte fácil para los educadores médicos adaptarse a esta forma pedagógica, pero los progresos en la educación médica, en conjunto a una asistencia sanitaria que restringe la presencia del alumnado en centros de salud, son las fuerzas motrices que impulsan al desarrollo de formas más eficientes y menos costosas de educación ${ }^{5}$.

En el caso de la Escuela de Medicina de la Pontificia Universidad Católica de Chile (PUC) la semiología se aprende formalmente en el curso de Integrado de Clínicas II para alumnos de tercer año. Este curso incluye en su programa clases lectivas, talleres prácticos de examen físico con fantomas y recursos interactivos, además de 
actividades prácticas hospitalarias con pacientes reales. Los alumnos son divididos en grupos de 5 a 6 personas, los cuales rotan tanto por actividades prácticas hospitalarias como por talleres de semiología presenciales con simulación a cargo de un tutor, realizándose estos últimos una vez por semana hasta completar un total de catorce talleres, que incluyen: examen físico general, examen abdominal, examen pulmonar, examen cardíaco, examen neurológico, examen articular, examen urológico, examen de mama, examen dermatológico, examen pediátrico, examen geriátrico, examen otorrinolaringológico, examen oftalmológico y radiografía de tórax.

Con el objetivo de facilitar el aprendizaje de habilidades prácticas semiológicas y de interpretación clínica básica por parte de los alumnos de tercer año de medicina, apoyando a su vez los talleres de simulación mencionados y enmarcados en la necesidad de mejorar el aprendizaje en tiempo y calidad por la nueva reforma curricular de nuestra escuela de medicina adoptada a partir del año $2015^{6}$, se implementó una plataforma virtual de aprendizaje y evaluación, disponible en la dirección: www.semiologia.cl, web interactiva de semiología médica, diseñada acorde los objetivos del curso y con material audiovisual, donde el alumno cuenta con material interactivo que logra agrupar y presentar la experiencia clínica en un formato flexible y amable, aprovechando recursos audiovisuales.

Circunscritos por una política de cambios curriculares iniciada hace décadas por parte de la Escuela de Medicina ${ }^{7}$ y al alero de los principios FAIR, planteados para lograr un mayor aprendizaje efectivo (entrega de retroalimentación, aprendizaje activo, individualizado y relevante) la realización de la intervención mencionada busca reforzar el aprendizaje activo del alumnado, donde es el estudiante el protagonista y responsable de la exploración y obtención del material de la plataforma. Asimismo, y siguiendo el paradigma nuevo de evaluación para el aprendizaje (en lugar de evaluación por el aprendizaje) se procura la entrega de retroalimentación efectiva e individualizado para optimizar su aprendizaje ${ }^{8}$.

\section{Metodología}

Se diseñó una página web que contiene, imágenes, vídeos y casos clínicos virtuales que incluían: historia clínica, examen físico y exámenes complementarios. Este material se encuentra distribuido en un total de 9 módulos que incluyen: examen físico general, examen físico abdominal, radiografía de tórax, examen dermatológico, examen neurológico, examen articular, examen pulmonar, examen cardiaco y pediatría. La organización y contenido de cada módulo se detallan en la Tabla 1. La web fue utilizada en el curso de semiología de tercer año (Integrados Clínica II) del año 2015.

A modo de ejemplo, las Figuras 1 y 2 corresponden a capturas de pantalla de los módulos de Examen abdominal y Radiografía de tórax respectivamente.

Como método de autoevaluación del aprendizaje logrado en cada uno de los talleres de simulación recién descritos, junto a la disponibilidad de la web interactiva para el estudio personal y de reforzamiento, se generó un banco de preguntas estructuradas que evaluaran el aprendizaje de los talleres prácticos simulados. Al finalizar cada taller de simulación, considerando la disponibilidad permanente de la web, se aplicó mediante la aplicación gratuita Edmodo 2016@ (disponible para computadores y dispositivos móviles), un minitest de 10 preguntas que evalúan el logro de objetivos mínimos para cada taller. Esta aplicación, se comporta como una red social que permite hacer llegar notificaciones o minitest a los alumnos por grupos específicos y asignarles tareas por separado, pudiendo realizar evaluaciones de desarrollo o selección múltiple, en un período de tiempo determinado, que en este curso fue de siete días, además se realiza retroalimentación instantánea con nota formativa de los mismos minitest, enviándolos de manera privada al correo electrónico de cada alumno.

Con el objetivo de evaluar la satisfacción global de los alumnos con los módulos en línea de autoinstrucción, se realizó un estudio observacional antes y después, el que consistió en realizar una encuesta (Tabla 2) al finalizar el curso de semiología, en la que se presentaron 7 aseveraciones, a la cuales los alumnos tenían que determinar su grado de acuerdo, siendo 1 muy de acuerdo y 4 muy en desacuerdo. Esta encuesta se realizó en base a los objeticos del curso de semiología y las recomendaciones realizadas por el Centro de Educación Médica de la Facultad de Medicina. Luego se realizó análisis de validez de contenido, muestreo y constructo mediante un análisis 


\section{Tabla 1. Módulos web y su contenido}

\begin{tabular}{|c|c|}
\hline Módulo & Organización \\
\hline Examen físico general & Imágenes y videos acompañados, si el caso lo amerita, de un breve texto explicativo \\
\hline Examen abdominal & $\begin{array}{l}\text { Dividido en: } \\
\text { a) Anatomía superficial: a su vez dividido en Referencias y Divisiones, ambos con animaciones } \\
\text { que el alumno explora para acceder al contenido } \\
\text { b) Inspección: Imágenes ilustrando los ítems Forma-Cicatrices-Hernias-Circulación colateral } \\
\text { c) Auscultación: imagen estática dotada de sonido. El alumno debe hacer "clic" y mantener pul- } \\
\text { sado (o mantener presionado en caso de usar smartphone) para escuchar el respectivo sonido, } \\
\text { simulando una auscultación. Incluye ruidos hidroaéreos normales y aumentados } \\
\text { d) Percusión: dividida en Normal y Matidez, con una imagen estática dotada de sonido. El } \\
\text { alumno debe hacer "clic" (o presionar en casos de usar smartphone/tablet) en la imagen para } \\
\text { escuchar el respectivo sonido, simulando una percusión } \\
\text { e) Palpación: videos breves explicativos del proceso de palpación en el examen físico abdominal }\end{array}$ \\
\hline Examen dermatológico & $\begin{array}{l}\text { Múltiples imágenes que muestran diversas lesiones de piel y fanéreos, distribuidas en los ítems } \\
\text { Color, Lesiones, Pelo y Uñas. Cada imagen es acompañada por un breve texto explicativo }\end{array}$ \\
\hline Examen neurológico & $\begin{array}{l}\text { Dividido en los ítems de: Conciencia y estado mental, en cuyo caso se incluyeron preguntas } \\
\text { de selección múltiple con respuesta y feedback inmediato; Parescraneanos, con videos explica- } \\
\text { tivos de su exploración y, en ciertos casos, lesiones características y sus manifestaciones clínicas; } \\
\text { Examen motor, con videos explicativos de su exploración cínica; Sensibilidad, con imágenes } \\
\text { y videos explicativos del proceso exploratorio y el sustrato anatomofisiológico subyacente, inclu- } \\
\text { yendo además síndromes sensitivos recurrentes en la práctica; Signos meníngeos, con videos } \\
\text { mostrando el método de exploración de éstos }\end{array}$ \\
\hline Examen articular & $\begin{array}{l}\text { Dividido en: Columna, Hombro, Codo, Muñeca, Mano, Cadera, Rodilla y Pies. Cada sub- } \\
\text { división cuenta con los ítems: Inspección, Movilidad y Palpación, en los que se distribuyen } \\
\text { imágenes y videos explicativos según sea el caso }\end{array}$ \\
\hline Examen pulmonar & $\begin{array}{l}\text { Se dispone de } 4 \text { casos clínicos, cada uno dividido en: } \\
\text { a) Historia: breve descripción del caso } \\
\text { b) Examen físico general: detalla las condiciones y hallazgos relevantes del examen general, } \\
\text { sean estos normales o patológicos } \\
\text { c) Examen pulmonar: dividido a su vez en Inspección, detallada en un texto breve; Palpación } \\
\text { acompañada también de un texto breve; Auscultación, donde el alumno debe presionar en una } \\
\text { imagen estática para obtener un sonido auscultatorio, emulando el proceso real; y Percusión, } \\
\text { similar a lo descrito anteriormente en el ítem de Examen abdominal } \\
\text { d) Radiografía: se cuenta con dos proyecciones: anteroposterior y lateral } \\
\text { e) Diagnóstico: el alumno obtiene la interpretación clínica de los ítems anteriores, elaborada } \\
\text { por el docente }\end{array}$ \\
\hline Examen cardíaco & $\begin{array}{l}3 \text { Casos clínicos, cada uno dividido en: } \\
\text { a) Historia: breve descripción del caso } \\
\text { b) Examen físico general: detalla las condiciones generales y datos relevantes del examen } \\
\text { general } \\
\text { c) Examen cardiaco: centrado en la Auscultación, cuyo proceso el alumno emula al presionar } \\
\text { sobre los } 5 \text { clásicos focos auscultatorios para así obtener el sonido respectivo } \\
\text { d) Examen complementario: siendo este un electrocardiograma relacionado al caso } \\
\text { e) Diagnóstico: donde el alumno obtiene la interpretación clínica de los ítems anteriores reali- } \\
\text { zada por el docente, por escrito }\end{array}$ \\
\hline Radiografía de tórax & $\begin{array}{l}\text { Módulo creado para facilitar la interpretación y análisis básico de este examen complementario. } \\
\text { Dividido a su vez en: } \\
\text { a) Normal: con los ítems de Técnica, donde se detallan y ejemplifican gráficamente los términos } \\
\text { de inclusión, rotación, penetrancia y posición; Hitos anatómicos, donde se presentan gráfi- } \\
\text { camente los hitos característicos a identificar en una radiografía } \\
\text { b) Alterada: donde se explican y presentan gráficamente radiografías patológicas como: } \\
\text { Condensación, Derrame pleural, Neumotórax, Nódulo pulmonar, Cardiomegalia, } \\
\text { Ensanchamiento mediastínico }\end{array}$ \\
\hline Pediatría & Videos explicativos que ilustran la medición de la talla y peso de un lactante \\
\hline
\end{tabular}



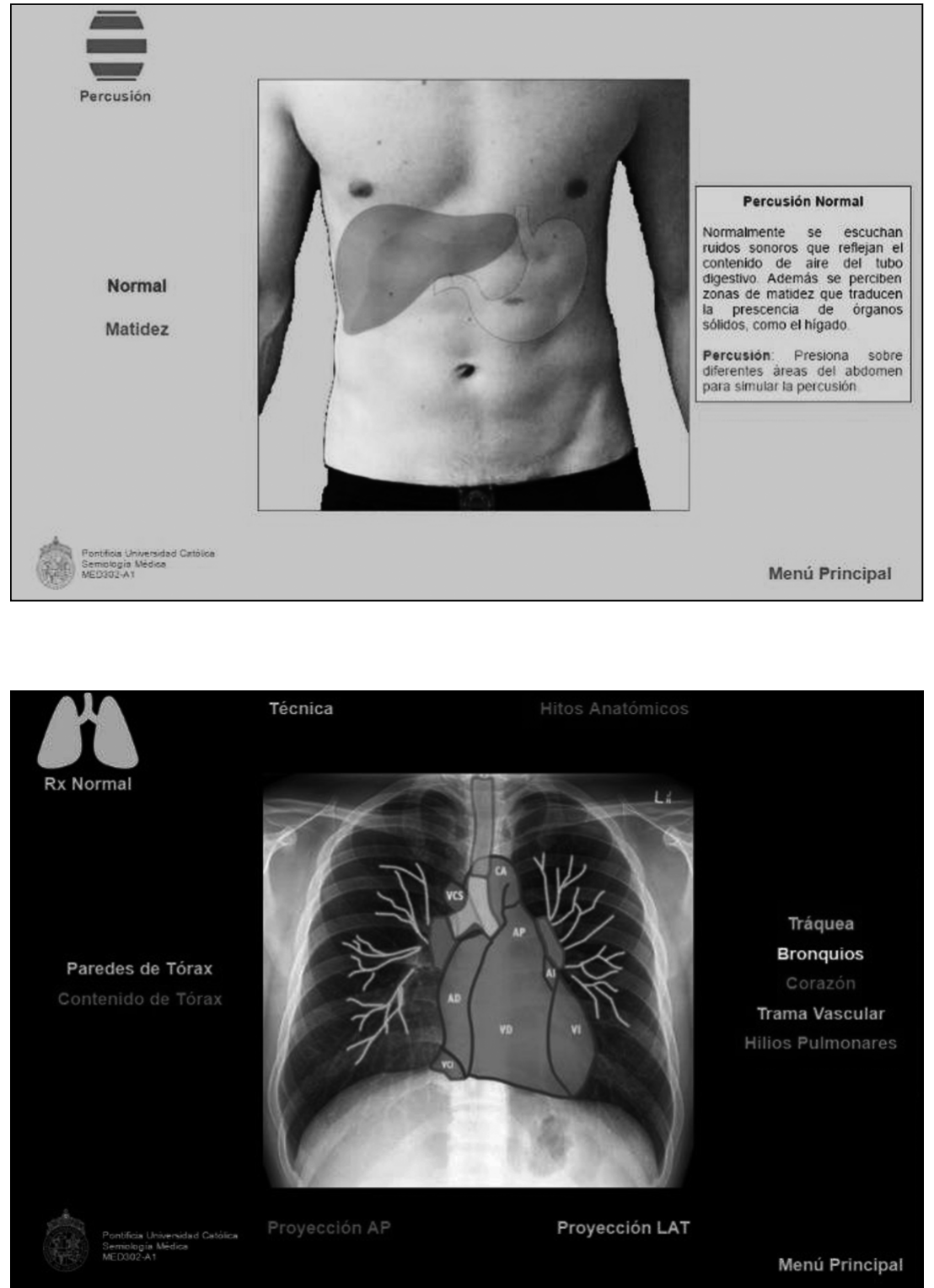

Figura 1. En esta sección se cuenta con un botón de Menú principal y 2 botones que direccionan a dos escenarios posibles: Matidez y Normalidad. En este caso, se muestra la situación Normal. El alumno debe hacer "click" o presionar en caso de estar en un smartphone - tablet sobre la proyección hepática, gástrica o la superficie abdominal para escuchar y evaluar la diferencia auditiva. Una experiencia similar se encuentra en la sección "Matidez".
Figura 2. En esta sección de la web correspondiente al módulo Radiografía de Tórax, los alumnos al posar el cursor sobre la placa encontrarán coloreados los elementos con su respectiva nominación. Al quitar el cursor de la imagen, la placa volverá a su original. factorial exploratorio con rotación Varimax. Los factores se seleccionaron de acuerdo al criterio de Kaiser-Guttman, que incluye a los factores con un auto-valor (eigenvalue) $>19$. También se utilizó el criterio de Cattell, que establece el punto de inflexión del grafico de sedimentación como valor de corte, considerando todos los factores sobre éste $^{9}$. Por otro lado, se midió la confiabilidad de los resultados de la encuesta en base al análisis de consistencia con alfa de Cronbach, que se expresa de 0 al $(<0,4$ : pobre, 0,4- < 0,6: moderada, 0,6 $<0,8$ : buena y 0,8 a 1 : excelente $)^{10}$.

Finalmente, se les pidió que le pusieran una nota al curso de 1 a 10 , siendo esta última la mejor nota. Para objetivar y cuantificar la utilidad de este nuevo método se procedió a comparar el promedio de notas del Examen Clínico Objetivo Estructurado (ECOE), que se realiza al finalizar el curso, con una población no expuesta a la intervención de los módulos de autoinstrucción, pero 
Tabla 2. Encuesta de satisfacción global

\begin{tabular}{|c|c|}
\hline Preguntas & Puntaje (1-4) \pm DE \\
\hline $\begin{array}{l}\text { 1. El contar con un taller online de autoaprendizaje facilitó mi aprendizaje de semiología para hacer } \\
\text { más eficiente la obtención de datos en la historia clínica }\end{array}$ & $1,76 \pm 0,85$ \\
\hline $\begin{array}{l}\text { 2. El contar con un taller online de autoaprendizaje facilitó mi aprendizaje de semiología en cuánto } \\
\text { a la interpretación de hallazgos relevantes el examen físico }\end{array}$ & $1,45 \pm 0,76$ \\
\hline 3. El feedback online respecto de las respuestas del test facilitó mi aprendizaje de semiología & $1,7 \pm 0,9$ \\
\hline $\begin{array}{l}\text { 4. El conocimiento adquirido en los talleres de autoaprendizaje fue útil para reforzar conocimientos } \\
\text { de semiología }\end{array}$ & $1,35 \pm 0,71$ \\
\hline 5. El conocimiento adquirido en los talleres de autoaprendizaje fue útil para motivarme a estudiar más & $1,82 \pm 0,86$ \\
\hline 6. Modificaría la metodología utilizada en los talleres de autoaprendizaje & $2,85 \pm 0,94$ \\
\hline 7. El conocimiento adquirido en los talleres de autoaprendizaje fue útil para rendir el OSCE & $1,55 \pm 0,81$ \\
\hline
\end{tabular}

DE: Desviación estándar.

con la misma exposición al resto de las instancias docentes y con un ECOE que contenía los mismos objetivos y el mismo nivel de dificultad, por lo que se eligió el curso del año previo.

Los análisis estadísticos se llevaron a cabo con el programa SPSS versión 17 . Un valor $\mathrm{p}<0,01$ se consideró significativo. Los datos son presentados en promedio \pm desviación estándar considerando un intervalo de confianza de 95\%. La comparación de ambas notas fue hecha con test $t$ de Student para variables continuas.

\section{Resultados}

Los resultados de esta experiencia de innovación incluyeron la percepción de los propios estudiantes y el impacto académico reflejado en las calificaciones finales.

El total de los alumnos de tercer año (n: 120) realizaron el módulo de auto instrucción online durante el segundo semestre del 2015 (53\% hombres, edad promedio 21 años), el 100\% respondió la encuesta al final del curso. Los resultados de la encuesta se encuentran en la Tabla 2. Todas las aseveraciones tuvieron un alto grado de acuerdo entre los estudiantes (entre 1,35 y 1,76), siendo la utilidad para reforzar conocimientos en semiología (pregunta 4) la aseveración con la que los alumnos mayor acuerdo tuvieron $(1,35 \pm 0,71)$. Ante la aseveración si modificarían la metodología utilizada en los módulos de autoinstrucción, hubo un alto grado de desacuerdo $(2,85 \pm 0,94)$.
Con respecto a la validez de constructo, el análisis factorial mostró que el instrumento posee una estructura de 2 factores, de acuerdo al criterio de auto-valor (eigenvalue) $>1$. El primer factor dio cuenta de $44,5 \%$ de la varianza, mientras que con los 2 factores en conjunto se explicó el 58,8\% de la varianza total. El primer factor contiene las preguntas 1, 2, 3, 4, 5 y 7 . El segundo contiene la pregunta 6.

Para evaluar la consistencia interna de la encuesta se calculó alfa de Cronbach, el cual resultó en 0,69 incluyendo todos los ítems y 0,79 excluyendo el ítem 6 . Ambos considerados buenos resultados.

Para evaluar la satisfacción global del módulo de autoinstrucción, se les solicitó que le pusieran una nota (de 1 a 10), cuya media fue de $8,3 \pm$ 1,38. Para evaluar la efectividad de los módulos de autoinstrucción se comparó la nota promedio de ECOE del año 2014 (media 5,53 $\pm 0,48$ ), en la que no se contó con los módulos online, con la del 2015 (media 5,93 $\pm 0,32$ ), resultando una diferencia significativa (valor $\mathrm{p}<0,0001$ ).

\section{Discusión}

La realización de los módulos interactivos online tuvo resultados promisorios en su impacto educacional.

Los módulos interactivos online tuvieron una buena evaluación por los alumnos, con una nota promedio de $8,3 \pm 1,38$. Por otro lado, los alumnos 
estuvieron de acuerdo en que el módulo de autoaprendizaje les fue útil en varios aspectos, como en la eficiente obtención de datos en la historia clínica, en la interpretación del examen físico, en la facilitación del aprendizaje y en el reforzamiento de conocimientos teóricos de semiología, lo cual era parte de los objetivos de la implementación de los módulos. Dentro de éstos, el mayor acuerdo se encontró en la aseveración que hacía referencia a la utilidad en reforzar "conocimientos de semiología”. Dicho hallazgo es interesante, ya que resalta el hecho de que los alumnos perciben los módulos en línea como una herramienta útil en apoyar los conocimientos teóricos de la medicina, lo que podría ser utilizado en el futuro para reemplazar parcialmente las clases lectivas o teóricas por clases en línea, reservando los espacios tradicionalmente ocupados como clases magistrales en actividades prácticas, actividades aplicadas o en la resolución de dudas con los docentes, haciendo más eficiente los espacios con los tutores.

Siguiendo con la línea de la importancia del aprendizaje activo y del alumno como protagonista de su conocimiento, es destacable que los alumnos estuvieron de acuerdo con que los módulos interactivos online los motivaron a estudiar más, lo que ciertamente es uno de los objetivos que se tienen como educador y lo que además es un objetivo de las escuelas de medicina en donde se busca que los alumnos desarrollen la capacidad de autoaprendizaje.

Otro punto importante es que los alumnos perciben que los módulos los ayudaron a rendir el ECOE, probablemente al poder clarificar dudas teóricas que no quedaron resueltas en los seminarios clásicos que les fueron dictados. Esta percepción se evidenció en una significativa mejoría que tuvo el promedio del curso en este examen con respecto al año anterior.

La novedad de la actividad online propuesta en el curso Integrado II y el impacto educacional de la misma podría sustentarse en 3 puntos.

El primero sería la entrega inmediata de retroalimentación, lo que permite que el alumno reconozca inmediatamente sus fortalezas y debilidades, pudiendo optimizar su estudio personal.

El segundo punto es la presentación de situaciones clínicas que incluyen los contenidos mínimos esperados para un alumno de tercer año de medicina, a las cuales no siempre se pueden exponer en sus rotaciones prácticas, dada la hete- rogeneidad de pacientes a los que son expuestos y de los tutores que están a cargo de los grupos.

Lo tercero sería la metodología interactiva con alto grado de apoyo audiovisual, lo que ciertamente hace que el aprendizaje de la semiología sea más atractivo y tangible ${ }^{11}$.

Estos 3 puntos podrían facilitar la estandarización de la enseñanza de la semiología en particular y de la medicina en general, la que frecuentemente está sesgada por problemas como heterogeneidad de docentes, exposición a diferentes situaciones clínicas, elementos que históricamente ocurren en la enseñanza de la medicina ${ }^{12}$.

Finalmente se puede decir que, si bien existe satisfacción dentro del alumnado con respecto a esta metodología, ésta aún se puede seguir perfeccionando, dado que pese a existir una tendencia hacia no modificar la metodología $(2,85 \pm 0,94)$, ésta no es absoluta. Esto da espacio al perfeccionamiento y mejoría del método. Por lo mismo es importante enfatizar que cualquier nueva metodología que se implemente, por más exitosa que sea inicialmente, tiene que estar constantemente retroalimentándose de sus usuarios, para actualizarse, perfeccionarse y adecuarse a las necesidades de las nuevas generaciones de futuros médicos.

Es importante mencionar que evaluando la validez y consistencia de la encuesta llama la atención que tanto el análisis factorial como el alfa de Cronbach indican fuertemente que habría que excluir la pregunta número 6 . El análisis factorial indica que hay 2 factores en la encuesta, siendo que el segundo sólo incluye la pregunta 6 y el alfa de Cronbach sube, incluyendo todos los ítems de la encuesta y excluyendo el ítem 6 , de 0,69 a 0,79 . Sin embargo, si bien al excluir el ítem 6 se aumenta la validez de constructo, ésta aún se mantiene en un rango considerado como "bueno". Además, al excluir ese ítem se pierde validez de contenido, dado que la percepción de los alumnos con respecto a la metodología utilizada (pregunta 6) es de gran valor para la evaluación del método, razón por la cual se decidió mantener.

Dentro de las limitaciones de este estudio cabe destacar la dificultad en la interpretación, ya que la nota del ECOE fue comparada con el curso del año previo y no con una población control dentro del mismo curso. La justificación es que los alumnos que no se expongan a la página web de semiología, podrían perjudicar su aprendizaje y calificación es frente a los compañeros que sí tuvieron exposición 
al material. Por lo que si bien, no es una población de control ideal, era la comparación más justa para los estudiantes.

En conclusión, podemos decir que los módulos interactivos en línea de semiología médica son una metodología bien evaluada por los alumnos, que posiblemente mejora su rendimiento y que, si bien puede ser perfeccionada, es una herramienta que se puede utilizar para mejorar el aprendizaje en cursos clínicos iniciales.

Agradecimientos: Agradecemos la valiosa colaboración de Arnoldo Riquelme y Oslando Padilla en el apoyo estadístico.

\section{Referencias}

1. Gazitúa R. Introducción a la semiología. En: Gazitúa R, Editores. Apuntes de semiología. Santiago, Chile: Pontificia Universidad Católica de Chile.

2. Nadler P, Gonzales R. Síntomas comunes. En: Mc Phee S, Papadakis M, Editores, Diagnóstico clínico y tratamiento $49^{\circ}$ edición. San Francisco, California: Appleton \& Lange: Mc GrawHill.; 2010. p. 35-75.

3. Jameson L. Introducción a la Medicina Clínica. En: Harrison Principios de Medicina Interna. 17ma. ed. México: Mc Graw-Hill Interamericana Editores; 2008. p. 2-28.
4. Reinoso T, Tamarit T, Pérez G. La formación de recursos humanos en salud necesarios para el mundo y los paradigmas vigentes. Educ Med Super 2012; 26 (4): 635-41.

5. Knutson D, Cain T, Hurtubise L, Kreger C. Lessons learned: developing e-learning to teach physical examination. The Clinical Teacher 2006; 3 (3): 163-9.

6. Cisternas M, Rivera S, Sirham M, Thone M, Valdés C, Pertuzé J, et al. Reforma Curricular de la Carrera de Medicina de la Pontificia Universidad Católica de Chile. Rev Med Chile 2016; 144: 102-7.

7. Sánchez I, Riquelme A, Moreno R, Mena B, Dagnino J, Grebe G. Revitalising medical education: the School of Medicine at the Pontificia Universidad Católica de Chile. The Clinical Teacher 2008; 5 (1): 57-61.

8. Schuwirth L, Van der Vleuten C.Programmatic assessment: From assessment of learning to assessment for learning. Med Teach 2011; 33 (6): 478-85.

9. Cattell RB. The scree test for a number of factors. Multivariate Behavioural Research 1966; 1: 245-76.

10. Cronbach L. Coefficient alpha and the internal structure of tests. Psychometrika 1951; 16: 297-334.

11. Cilliers F, Schuwirth L, Herman N, Adendorff H, van der Vleuten C. A model of the pre-assessment learning effects of summative assessment in medical education. Adv Health Sci Educ Theory Pract 2012; 17 (1): 39-53.

12. Harden R, Sowden S, Dunn W. Educational strategies in curriculum development: the SPICES model. Med Educ 1984; 18 (4): 284-97. 